\title{
CERTAIN CLASSES OF ANALYTIC AND MULTIVALENT FUNCTIONS
}

\author{
H. M. HOSSEN, H. M. SRIVASTAVA AND M. K. AOUF
}

Abstract. The main object of the present paper is to investigate the special classes

$$
\begin{gathered}
\mathcal{P}_{\alpha}^{*}(p, A, B) \text { and } \mathcal{R}_{\alpha}^{*}(p, A, B) \\
(0 \leq \alpha<p ;-1 \leq B<A \leq 1 ; p \in \mathbb{N}:=\{1,2,3, \ldots\})
\end{gathered}
$$

of analytic and $p$-valent functions in the open unit disk $\mathcal{U}$. In particular, various growth and distortion theorems, and several coefficient estimates, are obtained for these as well as related classes of analytic and $p$-valent functions in $\mathcal{U}$.

\section{Introduction and Definitions}

Let $\mathcal{S}(p)$ denote the class of functions $f(z)$ of the form:

$$
f(z)=z^{p}+\sum_{n=1}^{\infty} a_{p+n} z^{p+n} \quad(p \in \mathbb{N}:=\{1,2,3, \ldots\})
$$

which are analytic and $p$-valent in the open unit disk

$$
\mathcal{U}:=\{z: z \in \mathbb{C} \text { and }|z|<1\}
$$

We denote by $\Omega$ the class of bounded analytic functions $w(z)$ in $\mathcal{U}$ satisfying the conditions:

$$
w(0)=0 \text { and }|w(z)|<1 \quad(z \in \mathcal{U}) .
$$

A function $f(z)$ in $\mathcal{S}(p)$ is called $p$-valent starlike of order $\alpha$ in $\mathcal{U}$ if it satisfies the following conditions:

$$
\begin{aligned}
& \mathcal{R}\left\{\frac{z f^{\prime}(z)}{f(z)}\right\}>\alpha \text { and } \int_{0}^{2 \pi} \mathcal{R}\left\{\frac{z f^{\prime}(z)}{f(z)}\right\} d \theta=2 p \pi \quad\left(z=e^{i \theta}\right) \\
& (0 \leq \alpha<p ; p \in \mathbb{N} ; z \in \mathcal{U}) \text {. }
\end{aligned}
$$

Received November 19, 1997.

1991 Mathematics Subject Classification. Primary 30C45.

Key words and phrases. Analytic functions, multivalent functions, growth and distortion theorems, coefficient estimates, starlike functions, convex functions. 
We denote by $\mathcal{S}^{*}(p, \alpha)$ the class of all $p$-valent starlike functions of order $\alpha$ in $\mathcal{U}$. Further, a function $f(z)$ in $\mathcal{S}(p)$ is called $p$-valent convex of order $\alpha$ in $\mathcal{U}$ if it satisfies the following codnitions:

$$
\begin{array}{r}
\mathcal{R}\left\{1+\frac{z f^{\prime \prime}(z)}{f^{\prime}(z)}\right\}>\alpha \text { and } \int_{0}^{2 \pi} \mathcal{R}\left\{1+\frac{z f^{\prime \prime}(z)}{f^{\prime}(z)}\right\} d \theta=2 p \pi \quad\left(z=e^{i \theta}\right) \\
(0 \leq \alpha<p ; p \in \mathbb{N} ; z \in \mathcal{U}) .
\end{array}
$$

We donote by $\mathcal{K}(p, \alpha)$ the class of all $p$-valent convex functions of order $\alpha$ in $\mathcal{U}$. It follows from (1.2) and (1.3) that

$$
f(z) \in \mathcal{K}(p, \alpha) \Leftrightarrow \frac{z f^{\prime}(z)}{p} \in \mathcal{S}^{*}(p, \alpha)
$$

The class $\mathcal{S}^{*}(p, \alpha)$ was studied by Patil and Thakare [9] and the class $\mathcal{K}(p, \alpha)$ was considered by Owa [8].

Let $\mathcal{T}(p)$ denote the subclass of $\mathcal{S}(p)$ consisting of functions $f(z)$ of the form:

$$
f(z)=z^{p}-\sum_{n=1}^{\infty} a_{p+n} z^{p+n} \quad\left(a_{p+n} \geq 0 ; p \in \mathbb{N}\right) .
$$

We denote by $\mathcal{T}^{*}(p, \alpha)$ and $\mathcal{C}(p, \alpha)$ the classes obtained by taking intersections, respectively, of the classes $\mathcal{S}^{*}(p, \alpha)$ and $\mathcal{K}(p, \alpha)$ with $\mathcal{T}(p)$, that is,

$$
\mathcal{T}^{*}(p, \alpha)=\mathcal{S}^{*}(p, \alpha) \cap \mathcal{T}(p)
$$

and

$$
\mathcal{C}(p, \alpha)=\mathcal{K}(p, \alpha) \cap \mathcal{T}(p)
$$

The classes $\mathcal{T}^{*}(p, \alpha)$ and $\mathcal{C}(p, \alpha)$ were investigated by Owa [8], who proved the following results for these classes:

Lemama 1. Let the function $f(z)$ be defined by (1.5). Then $f(z)$ is in the class $\mathcal{T}^{*}(p, \alpha)$ if and only if

$$
\sum_{n=1}^{\infty}(p+n-\alpha) a_{p+n} \leq p-\alpha
$$

The result is sharp.

Lemma 2. Let the function $f(z)$ be defined by (1.5). Then $f(z)$ is in the class $C(p, \alpha)$ if and only if

$$
\sum_{n=1}^{\infty}\left(\frac{p+n}{p}\right)(p+n-\alpha) a_{p+n} \leq p-\alpha
$$

The result is sharp. 
Lemma 3. Let the function $f(z)$ defined by (1.5) be in the class $\mathcal{T}^{*}(p, \alpha)$. Then

$$
|z|^{p}-\frac{p-\alpha}{p+1-\alpha}|z|^{p+1} \leq|f(z)| \leq|z|^{p}+\frac{p-\alpha}{p+1-\alpha}|z|^{p+1}
$$

and

$$
p|z|^{p-1}-\frac{(p+1)(p-\alpha)}{p+1-\alpha}|z|^{p} \leq\left|f^{\prime}(z)\right| \leq p|z|^{p-1}+\frac{(p+1)(p-\alpha)}{p+1-\alpha}|z|^{p} .
$$

Each of these results is sharp.

Lemma 4. Let the function $f(z)$ defined by (1.5) be in the class $C(p, \alpha)$. Then

$$
|z|^{p}-\frac{p(p-\alpha)}{(p+1)(p+1-\alpha)}|z|^{p+1} \leq|f(z)| \leq|z|^{p}+\frac{p(p-\alpha)}{(p+1)(p+1-\alpha)}|z|^{p+1}
$$

and

$$
p|z|^{p-1}-\frac{p(p-\alpha)}{p+1-\alpha}|z|^{p} \leq\left|f^{\prime}(z)\right| \leq p|z|^{p-1}+\frac{p(p-\alpha)}{p+1-\alpha}|z|^{p} .
$$

Each of these results is sharp.

Let $\mathcal{P}_{\alpha}^{*}(p, A, B)$ denote the class of functions $f(z)$ of the form (1.1) which satisfy the condition:

$$
\frac{f(z)}{g(z)}=\frac{1+A \omega(z)}{1+B \omega(z)} \quad(\omega \in \Omega ;-1 \leq B<A \leq 1 ; z \in \mathcal{U})
$$

for a function

$$
g(z)=z^{p}-\sum_{n=1}^{\infty} b_{p+n} z^{p+n} \quad\left(b_{p+n} \geq 0 ; p \in \mathbb{N}\right)
$$

in the class $\mathcal{T}^{*}(p, \alpha)(0 \leq \alpha<p)$. Further, let $\mathcal{R}_{\alpha}^{*}(p, A, B)$ denote the class of functions $f(z)$ of the form (1.1) which satisfy the condition (1.14) for a function $g(z)$, defined by (1.17), but belonging to the class $C(p, \alpha)(0 \leq \alpha<p)$.

Clearly, we have

(i) $\mathcal{P}_{\alpha}^{*}(1, A, B)=\tilde{P}_{\alpha}(A, B)$ and $\mathcal{R}_{\alpha}^{*}(1, A, B)=\tilde{R}_{\alpha}(A, B)$ (Owa [5]; [7]);

(ii) $\mathcal{P}_{\alpha}^{*}(1, \beta,-\lambda \beta)=\tilde{S}_{\lambda}(\alpha, \beta)$ and $\mathcal{R}_{\alpha}^{*}(1, \beta,-\lambda \beta)=\tilde{C}_{\lambda}(\alpha, \beta)(0 \leq \alpha<1 ; 0<\beta \leq$ $1 ; 0 \leq \lambda \leq 1)$ (Owa [4], [6]);

(iii) $\mathcal{P}_{\alpha}^{*}(1,1,-\mu)=S_{\mu}(0, \alpha)(0 \leq \mu \leq 1)($ Altintas $[1])$.

\section{Growth and Distortion Theorems}

Theorem 1. Let the function $f(z)$ defined by (1.1) be in the class $\mathcal{P}_{\alpha}^{*}(p, A, B)$. Then

$$
\begin{aligned}
& \frac{(1-A|z|)\{(p+1-\alpha)-(p-\alpha)|z|\}|z|^{p}}{(1-B|z|)(p+1-\alpha)} \leq|f(z)| \\
\leq & \frac{(1+A|z|)\{(p+1-\alpha)+(p-\alpha)|z|\}|z|^{p}}{(1+B|z|)(p+1-\alpha)} \quad(z \in \mathcal{U}) .
\end{aligned}
$$


Each of these estimates is sharp.

Proof. We employ the technique used earlier by Goel and Sohi [2], and by Owa ([4] to [7]). Since $f(z) \in \mathcal{P}_{\alpha}^{*}(p, A, B)$, we have

$$
\frac{f(z)}{g(z)}=\frac{1+A \omega(z)}{1+B \omega(z)} \quad\left(\omega \in \Omega ;-1 \leq B<A \leq 1 ; z \in \mathcal{U} ; g \in \mathcal{T}^{*}(p, \alpha)\right) .
$$

Thus, by using Schwarz's lemma [3], we have $|\omega(z)| \leq|z|$ for $z \in \mathcal{U}$. After a simple computation, we find that

$$
\left|\frac{f(z)}{g(z)}-\frac{1-A B|z|^{2}}{1-B^{2}|z|^{2}}\right| \leq \frac{(A-B)|z|}{1-B^{2}|z|^{2}} \quad(z \in \mathcal{U}) .
$$

Consequently, we obtain

$$
\frac{1-A|z|}{1-B|z|} \leq\left|\frac{f(z)}{g(z)}\right| \leq \frac{1+A|z|}{1+B|z|} \quad(z \in \mathcal{U})
$$

which gives the inequalities in (2.1) with the aid of Lemma 3.

By taking

$$
\frac{f(z)}{g(z)}=\frac{1+A z}{1+B z}
$$

and

$$
g(z)=z^{p}-\frac{p-\alpha}{p+1-\alpha} z^{p+1}
$$

we can see that the estimates in (3.1) are sharp. This completes the proof of Theorem 1.

Theorem 2. Let the function $f(z)$ defined by (1.1) be in the class $\mathcal{R}_{\alpha}^{*}(p, A, B)$. Then

$$
\begin{aligned}
& \frac{(1-A(z))\{(p+1)(p+1-\alpha)-p(p-\alpha)|z|\}|z|^{p}}{(1-B|z|)(p+1)(p+1-\alpha)} \leq|f(z)| \\
\leq & \frac{(1+A(z))\{(p+1)(p+1-\alpha)+p(p-\alpha)|z|\}|z|^{p}}{(1+B|z|)(p+1)(p+1-\alpha)} \quad(z \in \mathcal{U}) .
\end{aligned}
$$

Each of these estimates is sharp.for

$$
\frac{f(z)}{g(z)}=\frac{1+A z}{1+B z}
$$

and

$$
g(z)=z^{p}-\frac{p(p-\alpha)}{(p+1)(p+1-\alpha)} z^{p+1} .
$$

The proof of Theorem 2 is completed by using the same technique as in the proof of Theorem 1 with the aid of Lemma 4. 
Theorem 3. Let the function $f(z)$ defined by (1.1) be in the class $\mathcal{P}_{\alpha}^{*}(p, A, B)$. Then

$$
\begin{aligned}
\left|f^{\prime}(z)\right| \leq & \frac{(1+A|z|)\{p(p+1-\alpha)+(p+1)(p-\alpha)|z|\}|z|^{p-1}}{(1+B|z|)(p+1-\alpha)} \\
& +\frac{(A-B)\{(p+1-\alpha)+(p-\alpha)|z|\}|z|^{p}}{(1+B|z|)^{2}\left(1-|z|^{2}\right)(p+1-\alpha)} \quad(z \in \mathcal{U}) .
\end{aligned}
$$

Proof. Since $f(z) \in \mathcal{P}_{\alpha}^{*}(p, A, B)$, by using (2.2), we obtain

$$
f^{\prime}(z)=\frac{1+A \omega(z)}{1+B \omega(z)} g^{\prime}(z)-\frac{(A-B) \omega^{\prime}(z)}{\{1+B \omega(z)\}^{2}} g(z) \quad(\omega \in \Omega) .
$$

We also have

$$
\left|\omega^{\prime}(z)\right| \leq \frac{1}{1-|z|^{2}} \quad(z \in \mathcal{U})
$$

by means of Carathéodory's theorem [3]. Hence we obtain Theorem 3 with the aid of Lemma 3.

Remark 1. We have not been able to obtain a sharp estimate for $\left|f^{\prime}(z)\right|$ for

$$
f(z) \in \mathcal{P}_{\alpha}^{*}(p, A, B) \text {. }
$$

Theorem 4. Let the function $f(z)$ defined by (1.1) be in the class $\mathcal{R}_{\alpha}^{*}(p, A, B)$. Then

$$
\begin{aligned}
\left|f^{\prime}(z)\right| \leq & \frac{p(1+A|z|)\{(p+1-\alpha)+(p-\alpha)|z|\}|z|^{p-1}}{(1+B|z|)(p+1-\alpha)} \\
& +\frac{(A-B)\{(p+1)(p+1-\alpha)+p(p-\alpha)|z|\}|z|^{p}}{(p+1)(1+B|z|)^{2}\left(1-|z|^{2}\right)(p+1-\alpha)} \quad(z \in \mathcal{U}) .
\end{aligned}
$$

The proof of Theorem 4 would make use of Lemma 4 in the same manner as we applied Lemma 3 in the proof of Theorem 3 .

Remark 2. We have not been able to obtain a sharp estimate for $\left|f^{\prime}(z)\right|$ for

$$
f(z) \in \mathcal{R}_{\alpha}^{*}(p, A, B)
$$

\section{A. Set of Coefficient Estimates}

Theorem 5. Let the function $f(z)$ defined by (1.1) be in the class $\mathcal{P}_{\alpha}^{*}(p, A, B)$. Then

$$
\left|a_{p+1}\right| \leq A-B+\frac{p-\alpha}{p+1-\alpha} .
$$

The estimate is sharp. Furthermore, for $B \geq 0$,

$$
\left|a_{p+2}\right| \leq(A-B) B+(A-B) \frac{p-\alpha}{p+1-\alpha}+\frac{p-\alpha}{p+2-\alpha} .
$$


Proof. Let

$$
\omega(z)=\sum_{n=1}^{\infty} c_{n} z^{n} \in \Omega .
$$

Then we obtain (cf. [3])

$$
\left|c_{1}\right| \leq 1 \text { and }\left|c_{2}\right| \leq 1-\left|c_{1}\right|^{2} .
$$

Since $f(z) \in \mathcal{P}_{\alpha}^{*}(p, A, B)$, by using (2.2), we have

$$
f(z)[1+B \omega(z)]=g(z)[1+A \omega(z)] \quad(\omega \in \Omega) .
$$

Then, on substituting into (3.5) the power series (1.1), (1.15), and (3.3) for the functions $f(z), g(z)$, and $\omega(z)$, respectively, we find that

$$
\begin{aligned}
& \left(z^{p}+\sum_{n=1}^{\infty} a_{p+n} z^{p+n}\right)\left(1+B \sum_{n=1}^{\infty} c_{n} z^{n}\right) \\
& =\left(z^{p}-\sum_{n=1}^{\infty} b_{p+n} z^{p+n}\right)\left(1+A \sum_{n=1}^{\infty} c_{n} z^{n}\right) .
\end{aligned}
$$

Equating coefficients of $z^{p+1}$ and $z^{p+2}$ on both sides of (3.6), we obtain

$$
a_{p+1}=(A-B) c_{1}-b_{p+1}
$$

and

$$
\begin{aligned}
a_{p+2} & =(A-B) c_{2}-A b_{p+1} c_{1}-B a_{p+1} c_{1}-b_{p+2} \\
& =(A-B) c_{2}-(A-B) b_{p+1} c_{1}-B(A-B) c_{1}^{2}-b_{p+2}
\end{aligned}
$$

Since $g(z) \in \mathcal{T}^{*}(p, \alpha)$, by using Lemma 1 , we have

$$
b_{p+1} \leq \frac{p-\alpha}{p+1-\alpha} \text { and } b_{p+2} \leq \frac{p-\alpha}{p+2-\alpha}
$$

which, in conjunction with (3.7) and (3.8), would lead us to the inequalities (3.1) and (3.2), respectively. The estimate for $\left|a_{p+1}\right|$ in (3.1) is sharp for

$$
\frac{f(z)}{g(z)}=\frac{1-A z}{1-B z}
$$

and

$$
g(z)=z^{p}-\frac{p-\alpha}{p+1-\alpha} z^{p+1} .
$$

Similarly, with the aid of Lemma 2, we can prove

Theorem 6. Let the function $f(z)$ defined by (1.1) be in the class $\mathcal{R}_{\alpha}^{*}(p, A, B)$. Then

$$
\left|a_{p+1}\right| \leq A-B+\frac{p(p-\alpha)}{(p+1)(p+1-\alpha)} .
$$


Furthermore, for $B \geq 0$,

$$
\left|a_{p+2}\right| \leq(A-B) B+(A-B) \frac{p(p-\alpha)}{(p+1)(p+1-\alpha)}+\frac{p(p-\alpha)}{(p+2)(p+2-\alpha)}
$$

The estimate for $\left|a_{p+1}\right|$ in (3.12) is sharp for

$$
\frac{f(z)}{g(z)}=\frac{1-A_{z}}{1-B_{z}}
$$

and

$$
g(z)=z^{p}-\frac{p(p-\alpha)}{(p+1)(p+1-\alpha)} z^{p+1}
$$

Theorem 7. Let the function $f(z)$ defined by $(1.1)$ be in the class $\mathcal{P}_{\alpha}^{*}(p, A, B)$. Then, for $B \geq 0$,

$$
\begin{aligned}
\mid a_{p+3} \leq & (A-B)\left(1+B+B^{2}\right)+\{A+B+(A-B) B\} \frac{p-\alpha}{p+1-\alpha} \\
& +(A+B) \frac{p-\alpha}{p+2-\alpha}+\frac{p-\alpha}{p+3-\alpha} .
\end{aligned}
$$

Proof. Equating the coefficients of $z^{p+3}$ on both sides of (3.6), we have

$$
\begin{aligned}
a_{p+3}= & -b_{p+3}+(A-B) c_{3}-\left(A b_{p+1}+B a_{p+1}\right) c_{2} \\
& -\left(A b_{p+2}-B a_{p+2}\right) c_{1} .
\end{aligned}
$$

Here, since

$$
c_{n}=\frac{1}{2 \pi i} \int_{|z|=r} \frac{\omega(z)}{z^{n+1}} d z \quad(0<r<1 ; n \in \mathbb{N})
$$

for the coefficients $c_{n}$ of an analytic function $\omega(z)$ in the open unit disk $\mathcal{U}$, we obtain

$$
\begin{aligned}
a_{p+3}= & -b_{p+3}+\frac{A-B}{2 \pi i} \int_{|z|=r} \frac{\omega(z)}{z^{4}} d z \\
& -\frac{A b_{p+1}+B a_{p+1}}{2 \pi i} \int_{|z|=r} \frac{\omega(z)}{z^{3}} d z-\frac{A b_{p+2}-B a_{p+2}}{2 \pi i} \int_{|z|=r} \frac{\omega(z)}{z^{2}} d z .
\end{aligned}
$$

Now, by using Schwarz's lemma [3], $|\omega(z)| \leq|z|$ for $z \in \mathcal{U}$. Therefore, we get

$$
\left|a_{p+3}\right| \leq\left|b_{p+3}\right|+\frac{(A-B)}{r^{3}}+\frac{A\left|b_{p+1}\right|+B\left|a_{p+1}\right|}{r^{2}}+\frac{A\left|b_{p+2}\right|+B\left|a_{p+2}\right|}{r} .
$$

Consequently, we have Theorem 7 with the aid of Lemma 1 and Theorem 5 .

Similarly, by applying Lemma 2 and Theorem 6, we have 
Theorem 8. Let the function $f(z)$ defined by (1.1) be in the class $\mathcal{R}_{\alpha}^{*}(p, A, B)$. Then, for $B \geq 0$,

$$
\begin{aligned}
\mid a_{p+3} \leq & (A-B)\left(1+B+B^{2}\right)+\{A+B+(A-B) B\} \frac{p(p-\alpha)}{(p+1)(p+1-\alpha)} \\
& +(A+B) \frac{p(p-\alpha)}{(p+2)(p+2-\alpha)}+\frac{p(p-\alpha)}{(p+3)(p+3-\alpha)} .
\end{aligned}
$$

Remark 3. We have not been able to obtain sharp estimates for

$$
\left|a_{p+n}\right| \quad(p \in \mathbb{N} ; n \in \mathbb{N} \backslash\{1\})
$$

for the function $f(z)$ belonging to the classes $\mathcal{P}_{\alpha}^{*}(p, A, B)$ and $\mathcal{R}_{\alpha}^{*}(p, A, B)$.

Theorem 9. Let the function $f(z)$ defined by (1.1) be in the class $\mathcal{P}_{\alpha}^{*}(p, A, 0)$. Then

$$
\left|a_{p+n}\right| \leq A\left(\frac{2 p+1-2 \alpha}{p+1-\alpha}\right)+\frac{p-\alpha}{p+n-\alpha} \quad(p \in \mathbb{N} ; n \in \mathbb{N}) .
$$

Proof. Since $f(z)$ belongs to the class $\mathcal{P}_{\alpha}^{*}(p, A, 0)$, from (3.6) we have

$$
\sum_{n=1}^{\infty}\left(a_{p+n}+b_{p+n}\right) z^{p+n}=A\left(z^{p}-\sum_{n=1}^{\infty} b_{p+n} z^{p+n}\right)\left(\sum_{n=1}^{\infty} c_{n} z^{n}\right) .
$$

Equating the coefficients of $z^{p+n}$ on both sides of (3.23), we have

$$
a_{p+n}+b_{p+n}=A\left(c_{n}-\sum_{m=1}^{n-1} b_{p+m} c_{n-m}\right)
$$

which, in view of (3.18), yields

$$
a_{p+n}+b_{p+n}=\frac{A}{2 \pi i} \int_{|z|=r} \frac{\omega(z)}{z^{n+1}}\left(1-\sum_{m=1}^{n-1} b_{p+m} z^{m}\right) d z .
$$

Consequently, by using Schwarz's lemma [3] once again, we find that

$$
\begin{aligned}
\left|a_{p+n}+b_{p+n}\right| & \leq \frac{A}{2 \pi} \int_{0}^{2 \pi} \frac{1}{r^{n}}\left|1-\sum_{m=1}^{n-1} b_{p+m} r^{m} c^{i m \theta}\right| d \theta \\
& \leq \frac{A}{2 \pi r^{n}} \int_{0}^{2 \pi}\left(1+\sum_{m=1}^{n-1} b_{p+m} r^{m}\right) d \theta \\
& =\frac{A}{r^{n}}\left(1+\sum_{m=1}^{n-1} b_{p+m} r^{m}\right) \\
& \leq \frac{A}{r^{n}}\left(1+\sum_{m=1}^{n-1} b_{p+m}\right) .
\end{aligned}
$$


Since (3.26) holds true for any $r(0<r<1)$ and since

$$
\sum_{m=1}^{n-1} b_{p+m} \leq \frac{p-\alpha}{p+1-\alpha} \quad(p \in \mathbb{N} ; n \in \mathbb{N})
$$

by Lemma 1, we have

$$
\left|a_{p+n}+b_{p+n}\right| \leq A\left(\frac{2 p+1-2 \alpha}{p+1-\alpha}\right)
$$

Hence we obtain

$$
\begin{aligned}
\left|a_{p+n}\right| & \leq\left|a_{p+n}+b_{p+n}\right|+\left|b_{p+n}\right| \\
& \leq A\left(\frac{2 p+1-2 \alpha}{p+1-\alpha}\right)+\frac{p-\alpha}{p+n-\alpha}
\end{aligned}
$$

because

$$
b_{p+n} \leq \frac{p-\alpha}{p+n-\alpha} \quad(p \in \mathbb{N} ; n \in \mathbb{N})
$$

again by Lemma 1 .

Similarly, with the aid of Lemma 2, we can prove

Theorem 10. Let the function $f(z)$ defined by $(1.1)$ be in the class $\mathcal{R}_{\alpha}^{*}(p, A, 0)$. Then

$$
\left|a_{p+n}\right| \leq A\left(\frac{2 p^{2}+2 p+1-(2 p+1) \alpha}{(p+1)(p+1-\alpha)}\right)+\frac{p(p-\alpha)}{(p+n)(p+n-\alpha)} \quad(p \in \mathbb{N} ; n \in \mathbb{N}) .
$$

Theorem 11. Let the function $f(z)$ defined by (1.1) be in the class $\mathcal{P}_{\alpha}^{*}(p, A, B)$ for $-1 \leq B<0,0<A \leq 1$, and $\Re\left(a_{p+n}\right) \geq 0(n=1, \ldots, k-p-1)$.

Then

$$
\left|a_{p+n}\right| \leq A-B+\frac{p-\alpha}{p+n-\alpha}(p \in \mathbb{N} ; c \in \mathbb{N}) .
$$

Proof. Since $f(z) \in \mathcal{P}_{\alpha}^{*}(p, A, B)$, from (3.6) we have

$$
\sum_{n=1}^{\infty}\left(a_{p+n}+b_{p+n}\right) z^{p+n}=\left[(A-B) z^{p}-\sum_{n=1}^{\infty}\left(B a_{p+n}+A b_{p+n}\right) z^{p+n}\right] \omega(z) .
$$

We thus find that

$$
\sum_{n=0}^{\infty}\left(a_{p+n}+b_{p+n}\right) z^{n}=-\sum_{n=0}^{\infty}\left[\left(B a_{p+n}+A b_{p+n}\right) z^{n}\right] \omega(z),
$$

where

$$
a_{p}=1, b_{p}=-1, \text { and } \omega(z)=\sum_{m=0}^{\infty} c_{m+1} z^{m+1}
$$


Equating the coefficients of $z^{m}$ on both sides of (3.34), we obtain

$$
a_{p+m}+b_{p+m}=-\sum_{n=0}^{m-1}\left(B a_{p+n}+A b_{p+n}\right) c_{m-n} .
$$

Hence, from (3.34) and (3.35), we find that

$$
\begin{aligned}
& \sum_{n=0}^{m}\left(a_{p+n}+b_{p+n}\right) z^{n}+\sum_{n=m+1}^{\infty} E_{n} z^{n} \\
& \quad=-\sum_{n=0}^{m-1}\left[\left(B a_{p+n}+A b_{p+n}\right) z^{n}\right] \omega(z) \quad(m \in \mathbb{N})
\end{aligned}
$$

for some coefficients $E_{n}$.

Using $|\omega(z)|<1$ for $z \in \mathcal{U}$ and Parseval's identity [3] on both sides of (3.36), we obtain

$$
\begin{aligned}
\sum_{n=0}^{m}\left|a_{p+n}+b_{p+n}\right|^{2} r^{2 n} & \leq \sum_{n=0}^{m}\left|a_{p+n}+b_{p+n}\right|^{2} r^{2 n}+\sum_{n=m+1}^{\infty}\left|E_{n}\right|^{2} r^{2 n} \\
& \leq \sum_{n=0}^{m-1}\left|B a_{p+n}+A b_{p+n}\right|^{2} r^{2 n}
\end{aligned}
$$

Letting $r \rightarrow \mathbb{1}$ in (3.37), we obtain

$$
\left|a_{p+m}+b_{p+m}\right|^{2} \leq \sum_{n=0}^{m-1}\left[\left|B a_{p+n}+A b_{p+n}\right|^{2}-\left|a_{p+n}+b_{p+n}\right|^{2}\right] .
$$

Setting $m=k-p$ in (3.38), we are led finally to the inequality:

$$
\begin{aligned}
\left|a_{k}+b_{k}\right|^{2} \leq & (A-B)^{2}-(1-B)^{2} \sum_{n=1}^{k-p-1}\left|a_{p+n}\right|^{2}-\left(1-A^{2}\right) \sum_{n=1}^{k-p-1}\left|b_{p+n}\right|^{2} \\
& -2(1-A B) \sum_{n=1}^{k-p-1} \Re\left(a_{p+n}\right) b_{p+n} \quad(k=p+1, p+2, p+3, \ldots) .
\end{aligned}
$$

Since $b_{p+n} \geq 0(p \in \mathbb{N} ; n \in \mathbb{N})$, making use of the inequalities assumed to hold true in Theorem 11, we find that

$$
\left|a_{k}+b_{k}\right| \leq A-B \quad(k=p+1, p+2, p+3, \ldots ; p \in \mathbb{N})
$$

that is, that

$$
\left|a_{p+n}+b_{p+n}\right| \leq A-B \quad(p \in \mathbb{N} ; n \in \mathbb{N})
$$


Hence, by using (3.30) and (3.41), we obtain

$$
\begin{aligned}
\left|a_{p+n}\right| & \leq\left|a_{p+n}+b_{p+n}\right|+\left|b_{p+n}\right| \\
& \leq A-B+\frac{p-\alpha}{p+n-\alpha} \quad(p \in \mathbb{N}, n \in \mathbb{N}) .
\end{aligned}
$$

This completes the proof of Theorem 11 .

Finally, the result of Theorem 11 is sharp for

$$
\frac{f(z)}{g(z)}=\frac{1-A z^{n}}{1-B z^{n}}
$$

and

$$
g(z)=z-\frac{p-\alpha}{p+n-\alpha} z^{p+n}
$$

Corollary 1 . Let the function $f(z)$ defined by $(1.1)$ be in the class $\mathcal{P}_{0}^{*}(p, A, B)$ for

$$
-1 \leq B<0,0<A \leq 1, \text { and } \Re\left(a_{p+n}\right) \geq 0 \quad(n=1, \ldots, k-p-1) .
$$

Then

$$
\left|a_{p+n}\right| \leq A-B+\frac{p}{p+n} \quad(p \in \mathbb{N} ; n \in \mathbb{N})
$$

The result is sharp.

Remark 4. Since

$$
\frac{p-\alpha}{p+n-\alpha}
$$

is decreasing in $n$, Theorem 11 gives us the inequality:

$$
\left|a_{p+n}\right| \leq A-B+\frac{p-\alpha}{p+1-\alpha} \quad(p \in \mathbb{N} ; n \in \mathbb{N})
$$

for $f(z) \in \mathcal{P}_{\alpha}^{*}(p, A, B)(-1 \leq B<0 ; 0<A \leq 1)$ satisfying the condition:

$$
\Re\left(a_{p+n}\right) \geq 0 \quad(n=1, \ldots, k-p-1) .
$$

With the aid of Lemma 2, we also have

Theorem 12. Let the function $f(z)$ defined by $(1: 1)$ be in the class $\Re_{\alpha}^{*}(p, A, B)$ for

$$
-1 \leq B<0,0<A \leq 1, \text { and } \Re\left(a_{p+n}\right) \geq 0 \quad(n=1, \ldots, k-p-1) .
$$

Then

$$
\left|a_{p+n}\right| \leq A-B+\frac{p(p-\alpha)}{(p+n)(p+n-\alpha)} \quad(p \in \mathbb{N} ; n \in \mathbb{N})
$$

The result is sharp for

$$
\frac{f(z)}{g(z)}=\frac{1-A z^{n}}{1-B z^{n}}
$$


and

$$
g(z)=z^{p}-\frac{p(p-\alpha)}{(p+n)(p+n-\alpha)} z^{p+n} .
$$

Corollary 2. Let the function $f(z)$ defined by (1.1) be in the class $\mathcal{R}_{0}^{*}(p, A, B)$, for

$$
-1 \leq B<0,0<A \leq 1 \text {, and } \Re\left(a_{p+n}\right) \geq 0 \quad(n=1, \ldots, k-p-1) .
$$

Then

$$
\left|a_{p+n}\right| \leq A-B+\frac{p^{2}}{(p+n)^{2}} \quad(p \in \mathbb{N} ; n \in \mathbb{N})
$$

The result is sharp.

\section{Acknowledgement}

The present investigation was supported, in part, by the Natural Sciences and Engineering Research Council of Canada under Grant OGP0007353.

\section{References}

[1] O. Altintas, "On the coefficients of certain analytic functions," Math. Japon., 33(1988), 653-659.

[2] R. M. Goel and N. S. Sohi, "On certain classes of analytic functions," Indian J. Pure Appl. Math., 11(1980), 1308-1324.

[3] Z. Nehari, Conformal Mapping, McGraw-Hill, New York, 1952.

[4] S. Owa, "On certain classes of analytic functions," Portugal. Math., 40(1981), 449-456.

[5] S. Owa, "A note on certain classes of analytic functions," Punjab Univ. J. Math. (Lahore), 14-15(1981/1982), 175-182.

[6] S. Owa, "A remark on certain classes of analytic function," Math. Japon., 28(1983), 15-20.

[7] S. Owa, "On certain subclasses of analytic functions," Bull. Calcutta Math. Soc., 77(1985), 25-33.

[8] S. Owa, "On certain classes of $p$-valent functions with negative coefficients," Simon Stevin, 59(1985), 385-402.

[9] D. A. Patil and N. K. Thakare, "On convex hulls and extreme points of $p$-valent starlike and convex classes with applications," Bull. Math. Soc. Sci. Math. R. S. Roumanie (N. S.), 27(75)(1983), 145-160.

Department of Mathematics, Faculty of Science, University of Mansoura, Mansours, Egypt.

E-mail: sinfac@eic.mans.eun.eg

Department of Mathematics and Statistics, University of Victoria, Victoria, British Columbia V8W 3P4, Canada.

E-mail: hmsri@uvvm.uvic.ca 